\title{
Design of Remote Virtual Experiment System of SCM Based on C/S
}

\author{
Chen Guangfeng ${ }^{a}$, Wang Linxia $^{b}$, Yang Xiangping $^{c}$, Cheng Siyuan $^{d}$ \\ School of Mechanical Engineering, Donghua University, Shanghai 201620, China.

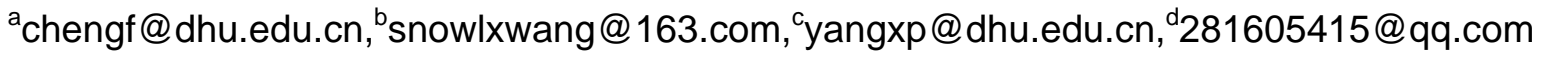

\begin{abstract}
Due to the limitation of laboratory equipment in SCM experiment teaching, this paper designs a $\mathrm{C} / \mathrm{S}$ based remote virtual experiment system for SCM education. The user enters the experimental information on the client experiment page. The virtual experiment server receives the experimental information and starts the virtual simulation software with the loading of simulation SCM model. The virtual simulation software sets parameters, and starts the simulation process through the virtual mouse and key operation. The server records the simulation processes and transfers to client page. The experimental results show that the experimental system has good effect in SCM experiment teaching.
\end{abstract}

Key words:SCM experiment teaching; virtual experiment system; simulation SCM model; C/S

\section{Introduction}

"Microcomputer principle and application", also known as "SCM", is a course with strong hands-on ability. It can cultivate students' ability of engineering design and application, engineering thinking and problem-solving ability ${ }^{[1]}$.

In the course of the teaching process, students often do experiments in the SCM Experimental box. However, due to the compression of class hours, and the lack of resources in many universities, students can only do some simple experiments in the laboratory, which is far from satisfying the goal of training students' product design ability ${ }^{[2]}$. The existing simulation softwares such as Proteus and Multisim provide some SCM simulation functions. Because of software authorization and the requirement of making simulation simulation models, it is not convenient for students to use, and it is not convenient for teachers to make statistical experiments. This paper develops a virtual SCM experiment system to solve these problems.

\section{General Design of Virtual Experiment System of SCM}

As shown in Figure 1, the C/S based virtual experiment system of SCM includes client, task scheduling server(TSS) and virtual experiment server(VES). All of the three key parts communicate through the Ethernet interface. 


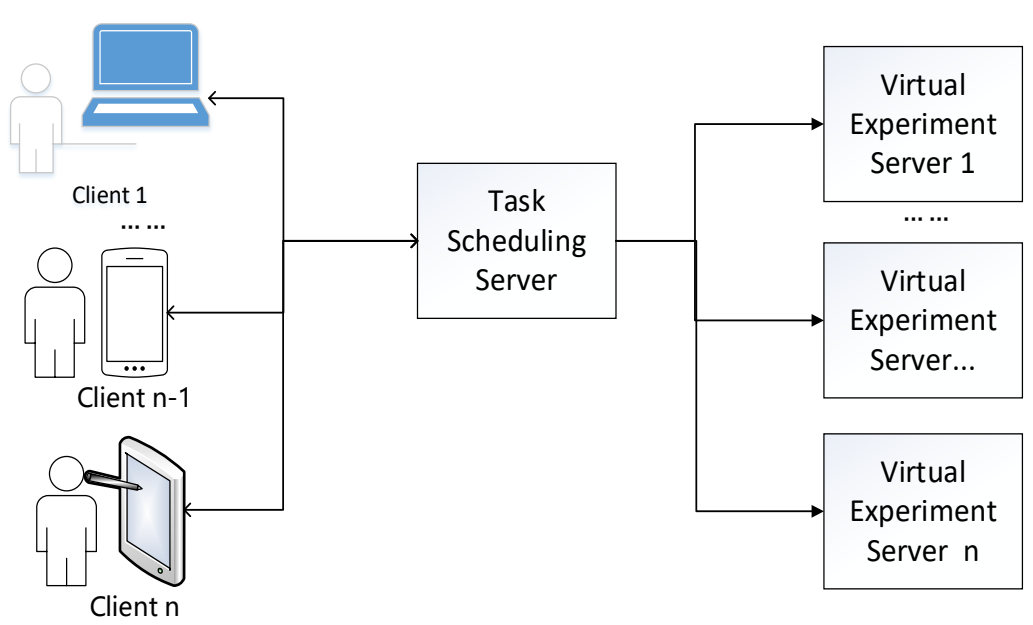

Fig.1 Schematic diagram of remote virtual experiment system of SCM

The client can be any intelligent device such as smart phones, computers, and tablets, etc. The user can start experimental software, input the experimental information and send the experimental informations to TSS.

Once the TSS obtained the experimental information from client, the simulation task is assigned to the idle virtual experiment server. If there were no idle virtual experiment server, it would feedback the client simulation task which is busy and prompt the waiting time which is needed. The virtual experiment server completes the simulation and generates video, which is fed back to the task scheduling server. The server records the above information and sends it back to the client, and the user can see the viedo of simulation result. TSS saves all experiment information for statistics and analysis. In accordance with the above ideas, the workflow is shown in Figure 2.

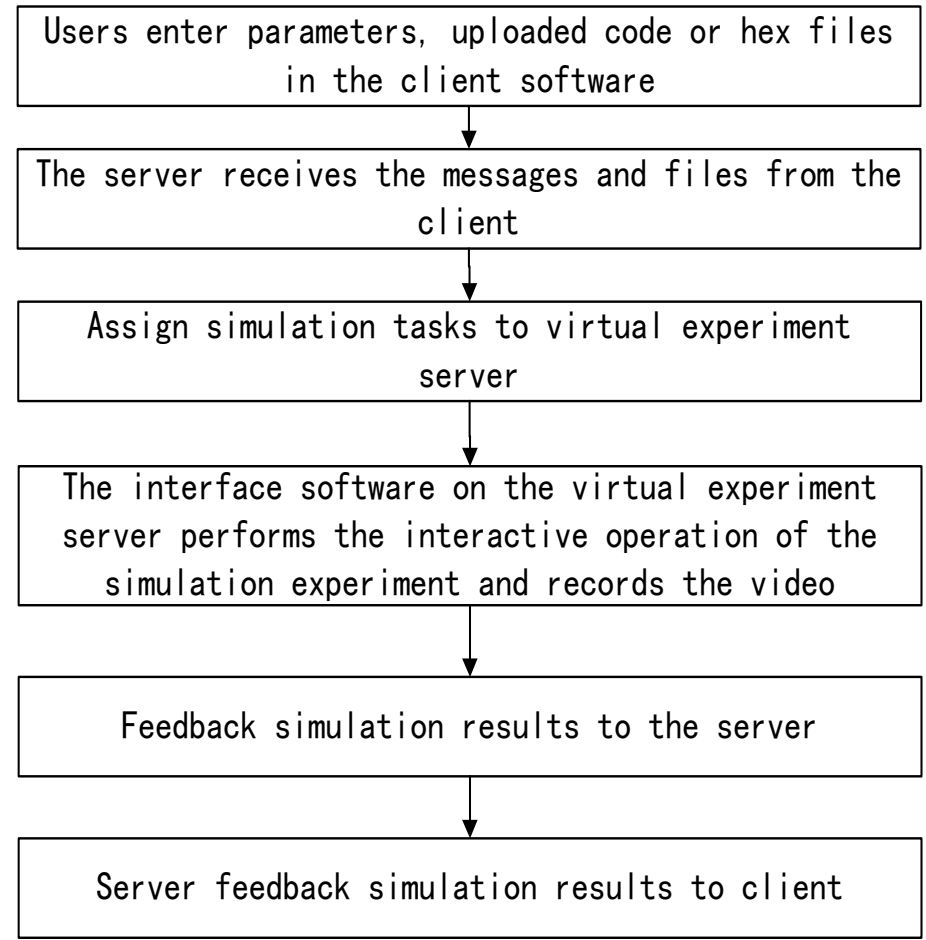

Fig.2 Workflow diagram

\section{Detail design of virtual experiment system of SCM}

\subsection{The Interface Design of the Client.}

The client software run on client device. Users enter the account password to log on to TSS. The interface of virtual experiment system pops up for users to input experimental information such as experimental content, experiment program and interaction operation in experiment process. The 
specific client interface is design as Figure 3. On the interface, the user selects the name of the experiment, then writes and upload program based on the experimental information, sets the simulation time and codes language on this interface .

Since many experiments need interactively operations in the process of experiment, an interaction openrtion settings page are provided as shown in Figure 3. By clicking "set up interactive operation", according to the corresponding information of simulation models and experimental summaries the page would popup. User can set the operation object, operation time, operation type and duration of interactive operation in the experimental model. According to the different operating objects and the corresponding experimental requirements, the operation types are divided into press, bounce, press up, adjusting the value and so on. After the interaction parameters are set, the user click "Add" to display the operating parameters in the interactive operation list. According to the different experimental models, the use adds the corresponding number of setting items. After all the interactive operations required for the experimental model are set, the user clicks "OK" to save the current interaction settings and submits the simulation task to task scheduling server.

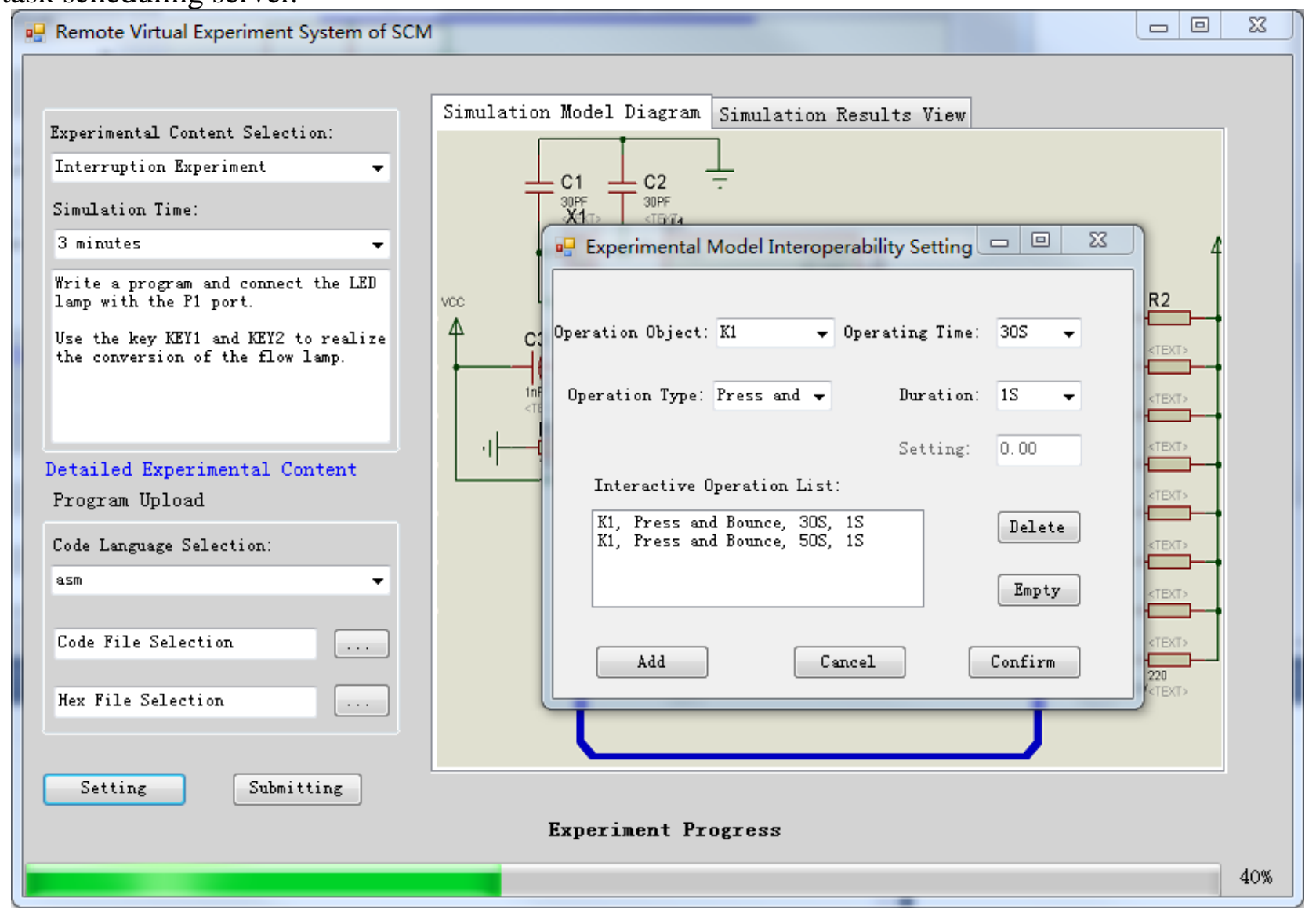

Figure 3 Client Interface Diagram

\subsection{Design of Task Scheduling Server.}

There is a task scheduling software developed in TSS. The main task of the task scheduling software is to verify user login and assign the simulation task. The TSS will check the avaliability of virtual experiment server, If all the virtual experiment servers are busy, the shortest waiting time is then feedback to the client. Otherwise, TSS will first store experimental information according to user number and date time. Store experimental information and transmit the received experimental information to the virtual experiment server.

Virtual Experiment Server Design. There are interface software and virtual simulation software are running on the virtual experiment server. Proteus which is a EDA tool software developed by Lab Center Electronics is selected as virtual simulation software Once the interface software 
receives the simulation task, and controls the Proteus to invoke the specified simulation model, and ensures that the Proteus Run at the front end of the interface, and loads the code file or the hex file for the simulation model.

If the user provide asm code file, the simulation software starts the code compilation. If the compilation error, it is feedback to the server and terminates the subsequent task.Interface software starts the video recording and the simulation on Proteus. While simulation starts, a timer will start timing. The interface software completes the action of virtual key according to the time point and operation type set by the interactive operation, and stops the simulation when the simulation time reaches the set point. Stop video recording, record users' submission of data and simulate video records. The simulation server simulation control process is shown in Figure 4.

\section{Demonstration of the virtual experiment system of SCM}

The user starts client software to login TSS, select "interrupt experiment (interrupt control lights)" experiment, the server provides the simulation model, experimental principle, interactive operation labels and software flow chart, and prompt users write the program A.asm according to the design hints. The simulation time is set 3 minutes, and the code language is "ASM". According to the simulation model diagram shown in Figure 3, during the experimental process the interactive operation is set. As shown in Figure 3, in the setting window of experimental model interactive operation, add operation object "K1". Operation type is "press and then release" and operation time and duration from the "30s" to "1s"; Add operation object "K2", and the type of operation is "press and then release". It continues from "50s" time is $1 \mathrm{s".} \mathrm{When} \mathrm{the} \mathrm{interaction} \mathrm{parameters} \mathrm{are} \mathrm{set}$ without error, the current interaction settings are cached. If the added parameter setting is wrong and needs to be modified, you can be deleted after the list is deleted and the interactive operation setting project is added. When end the interactive operations required for the experimental model are set up, click OK to cache the current interaction settings. Submit the simulation task.

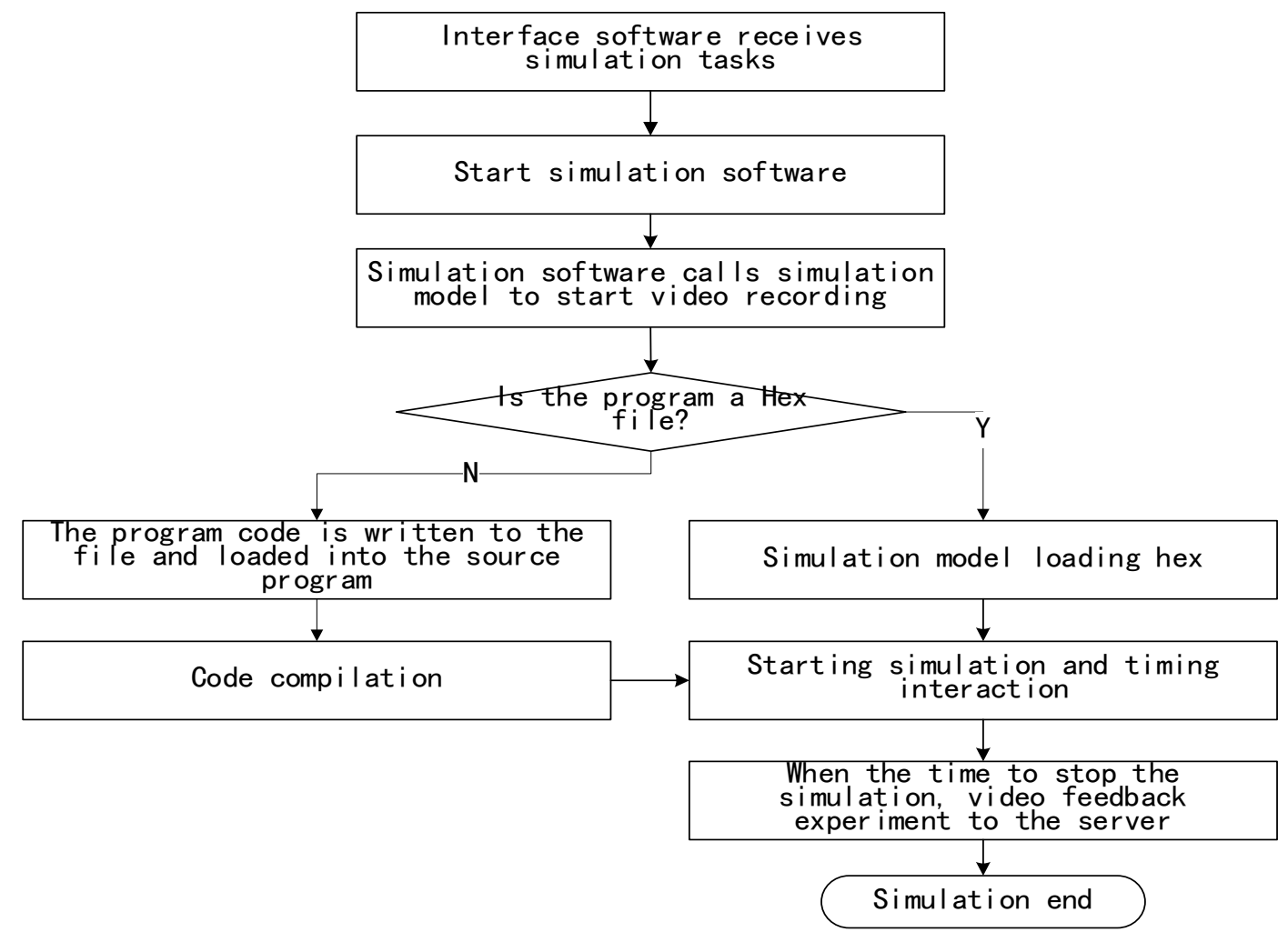

Fig.4 Virtual Experiment Simulation Flow Chart 
After receiving the A.asm file of the user through the Internet network, the server automatically stores it and transmits it to the virtual experiment server. The interface software of virtual experiment server receives the simulation task, and the interface software start video recording and real-time feedback to the client. If Proteus did not start, start it and ensure that it is in the forefront of the screen. Open the simulation model which is corresponding to "interrupt experiment (interrupt control lights)", and then the A.asm is specified as the code file of the simulation model, and the code is compiled, and the simulation and video recording will be stopped if errors occur. If there is no error, it starts the simulation and starts the timing. When the interface software reaches the set time of 30s, perform the set of interactive operation. Press the pop-up K1 button, the LED lamp right cycle. When the recording time reaches 50s, press the pop-up K1 button, the duration is 1s, and the LED lamp turns left. After 3 minutes of time, stop the simulation, turn off the Proteus and screen recording. The virtual experiment server caches the experimental video of the interrupted and the experiment (interrupt control flow lamp) on the server for two weeks, so as to let the client to check it again. The experimental feedback seen on the client side is shown in Figure 5.

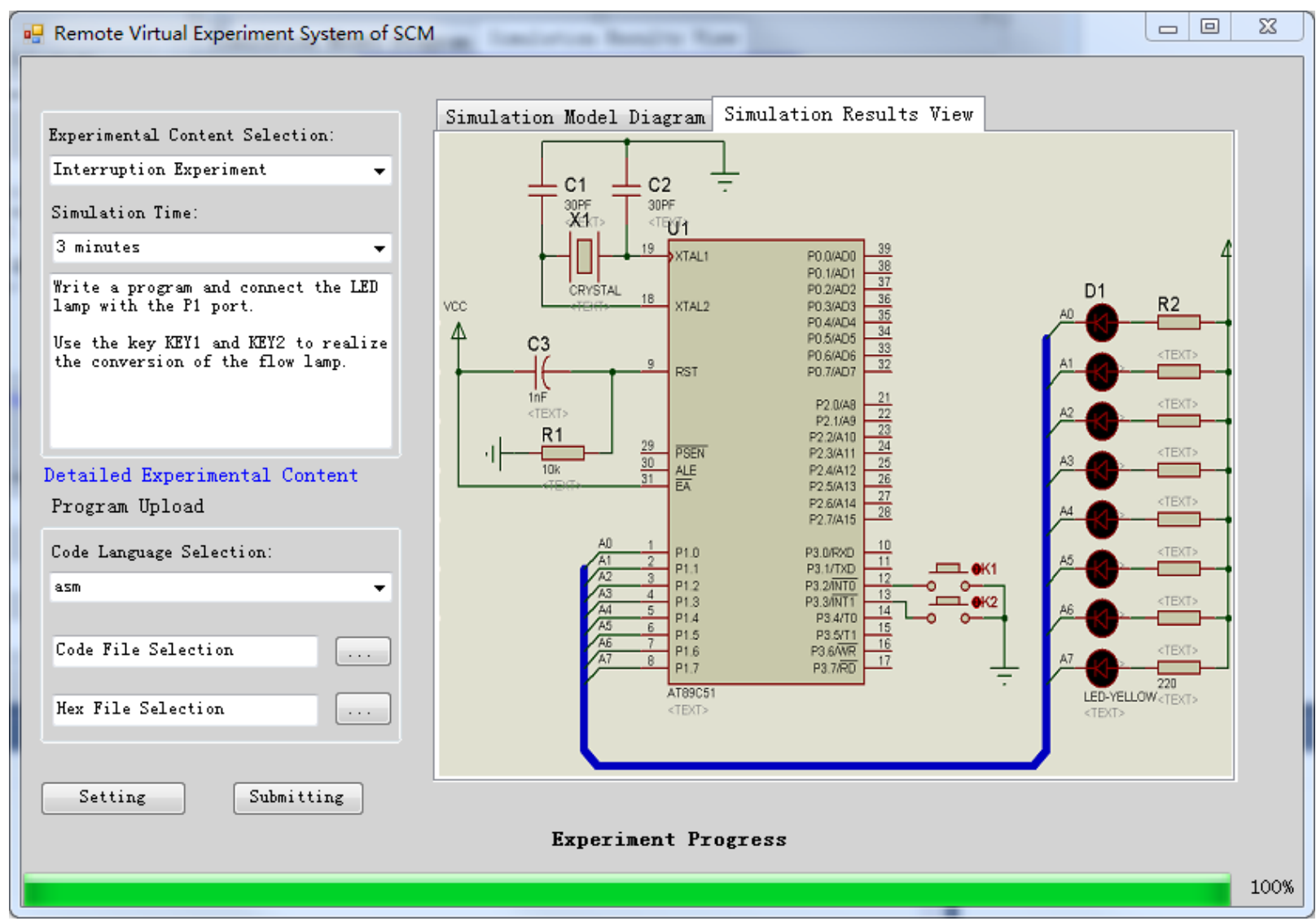

Fig. 5 Experimental Feedback

\section{Conclusion}

This paper proposed a virtual experiment system for SCM. The system is easy to use, flexible and diverse. It helps to cultivate students' innovative ability and the ability to use knowledge comprehensively. Meanwhile, it enables teachers to adjust experimental contents more conveniently, control experimental progress and improve teaching quality. 


\section{References}

[1] Zhu Jianguang, Zheng Hao, Liu Benwei. The Teaching Reform of SCM Principle and Interface Technology. Wuhan: Journal of Wuhan University,2012,58(S2):188-190.

[2] Ding Baohua, Zhang Youzhong, Chen Jun, Meng fan Xi. Experimental Teaching Reforms and Practices of MCU Principle and Interface. Experimental Technology and Management,2010,27(01): 117-119.

[3] Tian Feng. Research on Integration of Virtual Experiment and Real Experiment. Experimental Technology and Management,2005,22(11):89-92

[4] Zhou Lingbin, Zhang Jingwu. Teaching and Application Simulation of SCM in Proteus. Microcontrollers \& Embedded Systems,2008(1):76-79

[5] Ye Jianbo, Zhu Shuangdong. Virtual Simulation of SCM Based on Proteus. Informatization Research,2008,34(11):23-24,67.

[6] Zhang Yigang, Xiu Lincheng, Hu Zhenjiang. Application design of MCS-51. Harbin: Harbin Institute of Technology Press,1996. 